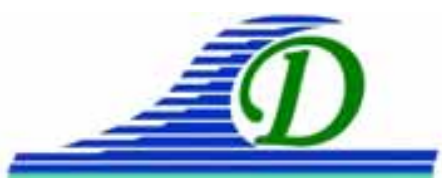
XIII ${ }^{\text {èmes }}$ Journées Nationales Génie Côtier - Génie Civil
Dunkerque, 2-4 juillet 2014

DOI:10.5150/jngcgc.2014.020 @ Editions Paralia CFL

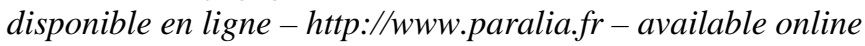

\title{
Variabilité longitudinale du jet de rive en présence de conditions dissipatives
}

\section{Nadia SENECHAL ${ }^{1}$, Giovanni COCO ${ }^{2}$, Nathaniel PLANT ${ }^{3}$, Karin BRYAN $^{4}$}

1. Université Bordeaux, UMR EPOC 5805, 33405 Talence, France.

n.senechal@epoc.u-bordeaux1.fr

2. Environmental Hydraulics Institute, "IH Cantabria", Universidad de Cantabria, Santander, Spain.

3. USGS, Saint Petersburg, USA.

4. Dept. of Earth and Ocean Sciences, University of Waikato, Hamilton, New Zealand.

\section{Résumé :}

Cette étude présente les résultats préliminaires de la variabilité longitudinale du jet de rive en présence de conditions dissipatives à partir de données vidéo. Les données présentées ici ont été acquises à marée basse sur une période de 4 heures en présence d'une plage présentant une structure morphologique complexe et en présence d'une houle longue énergétique ( $H s=1.9 \mathrm{~m})$. Les données indiquent que la hauteur associée au jet de rive est dominée par l'énergie contenue dans les basses fréquences et qu'elle varie significativement (facteur 1.56) sur une courte distance $(<250 \mathrm{~m}$ ) alors même que les conditions de houle en zone de surf, mesurées à environ $120 \mathrm{~m}$ de la position moyenne de la ligne d'eau ne présentent pas de variabilité longitudinale statistiquement significative. Les paramètres environnementaux habituellement utilisés pour paramétrer le jet de rive ne permettent pas à eux seuls d'expliquer cette variabilité longitudinale.

Mots-clés : Jet de rive, Infragravitaire, Gradient, Pente de plage.

\section{Abstract:}

Here we present preliminary results on alongshore variability of runup in presence of dissipative conditions. Video derived runup data were collected along four cross-shore transects spaning a 4-hour time window centered around low tide in presence of a long and energetic swell $(\mathrm{Hs}=1.9 \mathrm{~m})$. Data indicate that runup is dominated by infragravity energy and that runup elevations can vary by a factor 1.56 over a $250 \mathrm{~m}$ stretch of coast while inner surf zone wave height variability is not significant. The parameters commonly used to characterize runup can not explain this alongshore variability.

Keywords : Runup, Video survey, Nearshore bar, Alongshore variability.

\section{Introduction}

Le développement de stratégie de gestion du risque d'érosion et d'inondation marine en zone côtière, nécessite une bonne connaissance des processus naturels. Le jet de rive en est une composante clé puisqu'il délimite l'élévation maximale atteinte par la ligne d'eau et est l'un des vecteurs primordiaux du transfert sédimentaire entre le haut et le 
bas de plage. Sa paramétrisation est donc indispensable dans le dimensionnement des ouvrages côtiers. Il se décompose généralement en deux composantes : le set-up, qui correspond à une surévaluation de la position moyenne de la ligne d'eau associée au déferlement des vagues, et le run-up, fluctuation de cette ligne d'eau autour de la position moyenne. De nombreuses études ont été menées ces dernières années afin de paramétrer ce terme et notamment les travaux de STOCKDON et al. (2006) qui ont porté sur la synthèse de données acquises sur des sites contrastés. Il est généralement admis que la dynamique du jet de rive varie en fonction de paramètres hydrodynamiques (conditions de vagues) et environnementaux (pente de plage), paramétrés sous la forme du paramètre d'Irribaren :

$$
\xi_{0}=\frac{\tan \beta}{\left(H_{0} / L_{0}\right)^{1 / 2}}
$$

où $H_{0}$ et $L_{0}$ représentent respectivement la hauteur et la longueur d'onde des vagues au large, $\beta$ la pente de plage. Toutefois cette approche ne peut généralement pas s'appliquer à l'ensemble des composantes du jet de rive. Celui-ci est généralement subdivisé en une composante incidente associée aux fréquences des vagues incidentes et une composante infragravitaire (généralement associée à $f<0.05 \mathrm{~Hz}$ ). Lors de conditions dissipatives, la dynamique du jet de rive est généralement dominée par la composante infragravitaire du run-up (RUESSINK et al., 1998; RUGGIERO et al., 2004, SENECHAL et al., 2011a) alors que lors de conditions réflectives, la composante incidente $(0.05 \mathrm{~Hz}<f<0.25 \mathrm{~Hz}$ ) domine (GUZA \& THORNTON, 1982 ; STOCKDON et al., 2006). De nombreuses lacunes demeurent néanmoins sur la variabilité longitudinale du jet de rive en présence d'un même forçage. La plupart des études ont en effet été menées sur un seul profil de plage. RUGGIERO et al. (2004) avaient étudié la variabilité longitudinale mais uniquement en intégrant la pente de plage; les travaux récents de GUEDES et al. (2012) ont permis de montrer que des variations longitudinales du taux de dissipation des vagues et/ou la génération d’harmoniques pouvaient également expliquer la variabilité du jet de rive en présence d'une forçage modéré et de conditions réflectives.

Dans cette étude nous présentons les résultats préliminaires portant sur la variabilité longitudinale du jet de rive en présence d'une morphologie tri-dimensionnelle et de conditions dissipatives.

\section{Matériel et méthode}

Les données ont été acquises dans le cadre de la campagne internationale ECORS-Truc Vert 2008 (SENECHAL et al., 2011b). Le forçage au large a été obtenu à partir d'une bouée de houle directionnelle Datawell déployée au large de la zone d'étude par $20 \mathrm{~m}$ de fond environ en collaboration avec le SHOM. Compte tenu de la tridimensionnalité de la bathymétrie liée à la présence de deux systèmes de barres sableuses littorales 


\section{XIII ${ }^{\text {èmes }}$ Journées Nationales Génie Côtier - Génie Civil \\ Dunkerque, 2-4 juillet 2014}

(figure 1 à gauche, on peut voir la barre interne), des capteurs de pression, notés PUV dans la suite, ont également été déployés sur la barre interne en collaboration avec la NPS-USA (figure 1, croix rouges). Les données de jet de rive ont été extraites des images vidéo acquises à $2 \mathrm{~Hz}$ durant toute la période de la campagne de terrain en s’appuyant sur la méthode utilisée dans Sénéchal et al. (2011a). Pour cela des lignes de pixels perpendiculaires à la plage (transects) ont été extraites à plusieurs positions longitudinales de la plage (figure 1, en haut à droite). A partir de ces images, la position instantanée de la ligne d'eau a été digitalisée. Connaissant l'altitude du plan d'eau, la topographie de la plage et les corrections géométriques des caméras, les positions ont ensuite été converties en élévation (figure 1 en bas à droite). Par cette méthode il est possible de reconstruire des signaux d'élévation du jet de rive.
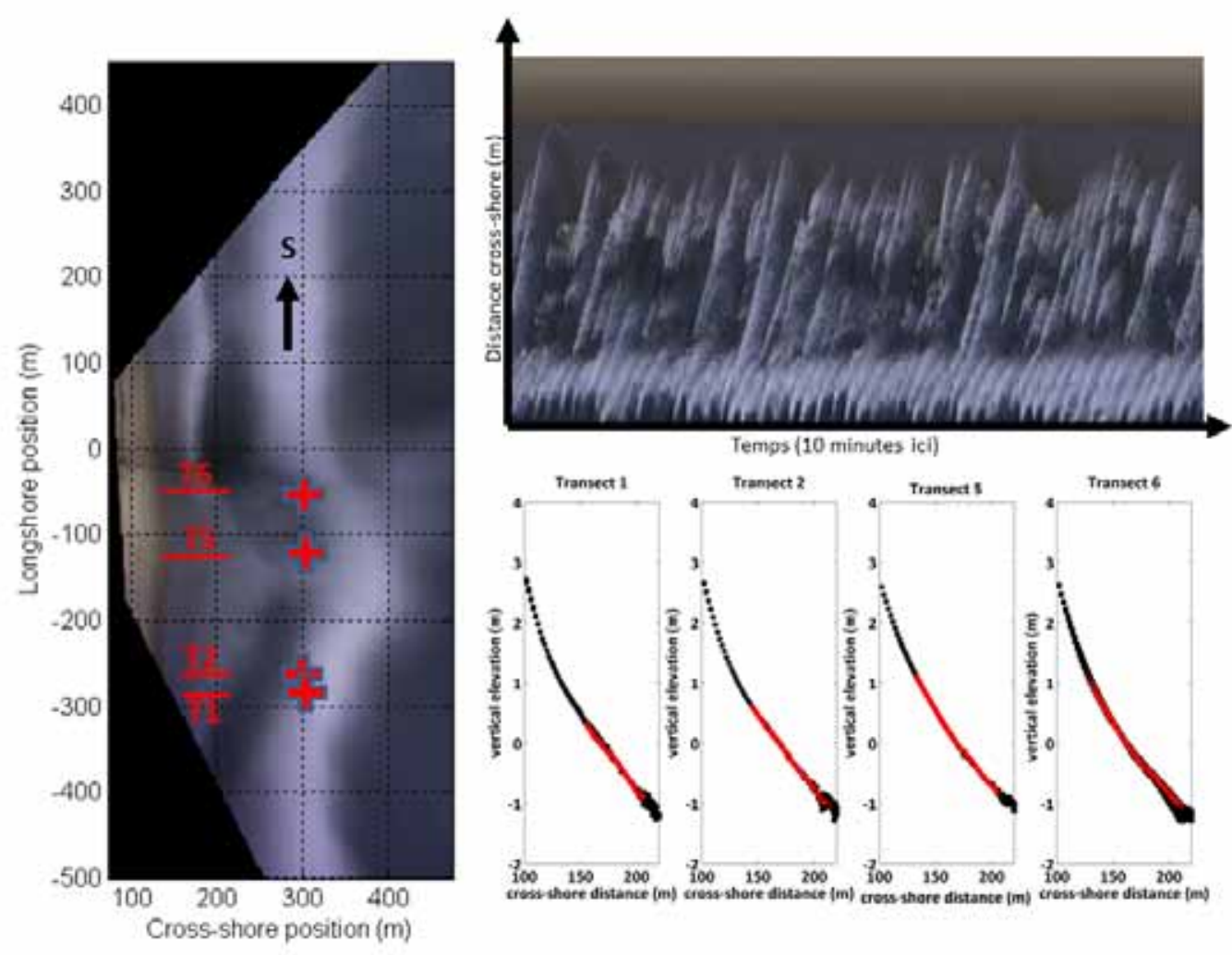

Figure 1. Image redressée et rectifiée de la zone d'étude avec emplacement des transects et des capteurs de pression. Exemple de timestacks permettant l'extraction de l'altitude de la ligne d'eau et du jet de rive. Profil des transects (noir) et excursion du jet de rive (rouge). Les distances sont données à partir du système vidéo situé sur la dune pré-littorale.

Les signaux de jet de rive et des capteurs de pression ont ensuite été analysés en utilisant la même méthodologie. Les hauteurs associées au jet de rive et aux vagues dans la zone de surf ont été estimées par intégration du spectre d'élévation (équation 2) par 
pas de 15 minutes dans les différentes bandes de fréquence afin de déterminer les composantes incidente $(0.05 \mathrm{~Hz}<f<0.24 \mathrm{~Hz})$ et infragravitaire $(0.004 \mathrm{~Hz}<f<0.05 \mathrm{~Hz})$.

$$
R=4 * \sqrt{\int_{f_{1}}^{f_{2}} P S D(f) d f}
$$

où $R$ représente la hauteur associée au jet de rive ou aux vagues en zone de surf, et PSD représente le spectre de densité d'énergie du signal de jet de rive ou des capteurs de pression. La pente de plage, en accord avec les données de la littérature (RUGGIERO et al., 2004 ; STOCKDON et al., 2006 ; SENECHAL et al., 2011a ; GUEDES et al., 2012), a été estimée à partir de la pente linéaire calculée sur une zone définie par \pm deux fois l'écart type de l'excursion horizontale du jet de rive.

Les données présentées dans cette étude concernent quatre transects extraits à marée basse en mortes eaux sur une période de 4 heures. Ce choix a été motivé par la recherche de conditions de forçage (vagues et morphologie) aussi stationnaires que possible et sur une durée suffisamment longue. Au cours de la période retenue, les variations d'altitude du plan d'eau moyen ont été inférieures à 0.4 m.

\section{Résultats et discussion}

\subsection{Conditions environnementales}

La figure 2 représente les conditions environnementales pendant la période d'étude. La hauteur des vagues au large mesurée par $20 \mathrm{~m}$ de fond est restée relativement stationnaire, variant entre $1.75 \mathrm{~m}$ et $1.95 \mathrm{~m}$ soit une variation maximale de $10 \%$ entre le minimum et le maximum. De la même façon, la période n'a que peu varié (de l’ordre de 15\%) s'échelonnant entre 12 s et 14 s. L’incidence est restée Nord pendant toute la période. Les valeurs calculées du paramètre d’Iribarren (équation1) sont généralement faibles et inférieures à 0.3 ; ces valeurs caractérisent généralement des jets de rive dissipatifs (RUESSINK et al., 1998 ; STOCKDON et al., 2006) dominés par la composante infragravitaire.

\subsection{Spectre de jet de rive et en zone de surf}

La figure 3 représente les spectres moyens calculés au niveau des capteurs de pression (PUV) et du jet de rive (Transect). La moyenne a été calculée à partir des 17 fenêtres temporelles. Chaque spectre moyen possède donc 34 degrés de liberté. La barre verticale continue représente la fréquence de coupure entre bande incidente et bande infragravitaire $(f=0.05 \mathrm{~Hz}$ ), la droite en pointillés représente la fréquence pic au large. On distingue au niveau des capteurs PUV situés en zone de surf la présence du pic de houle incident, en particulier au niveau des capteurs PUV1 et PUV2. Le spectre présente également une structure bimodale dans la bande infragravitaire. A l'inverse, les spectres calculés dans la zone de jet de rive ne présentent pas de pics statistiquement 


\section{XIII ${ }^{\text {èmes }}$ Journées Nationales Génie Côtier - Génie Civil \\ Dunkerque, 2-4 juillet 2014}

significatifs dans la bande incidente ni dans la bande infragravitaire et la zone de saturation d'énergie s’étend jusque dans les fréquences infragravitaires.
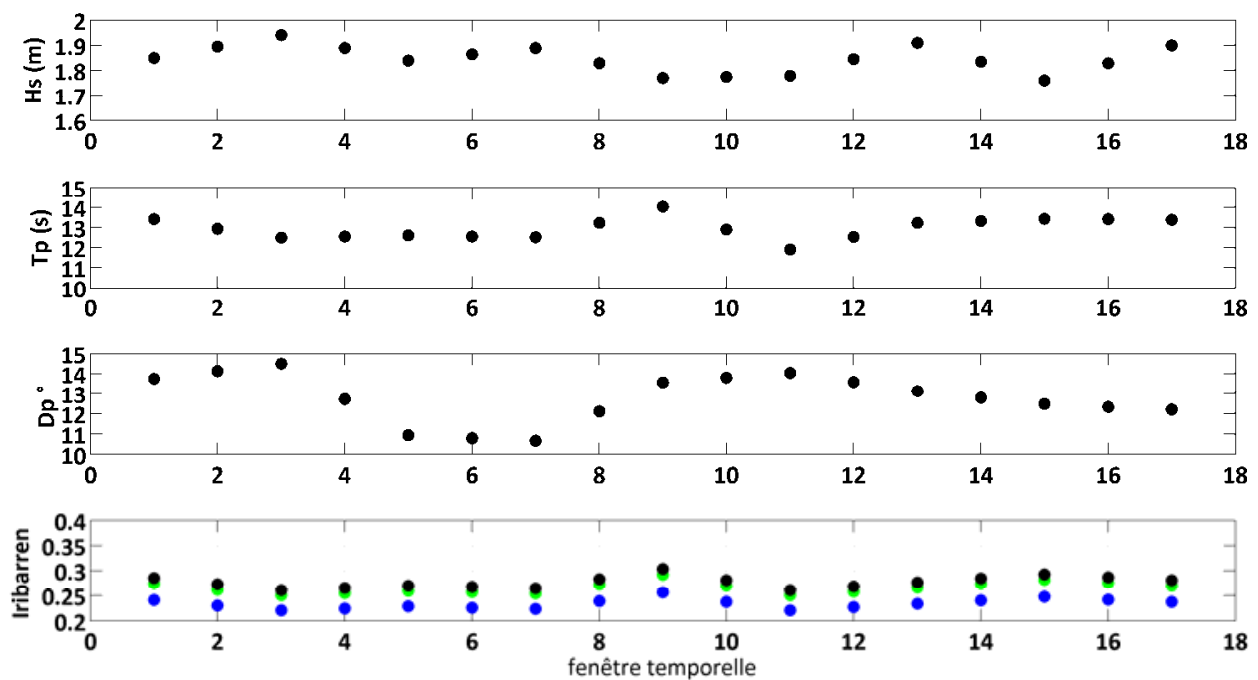

Figure 2. Conditions environnementales pendant les 4 heures de données représentant

la hauteur significative des vagues au large Hs (par $20 \mathrm{~m}$ de fond), la période pic associée, la direction pic par rapport à la normale (positif lorsque venant du Nord) et le paramètre d'Iribarren calculé au niveau des différents transects (en bleu T1 et T2, en vert $T 5$ et en noir T6).
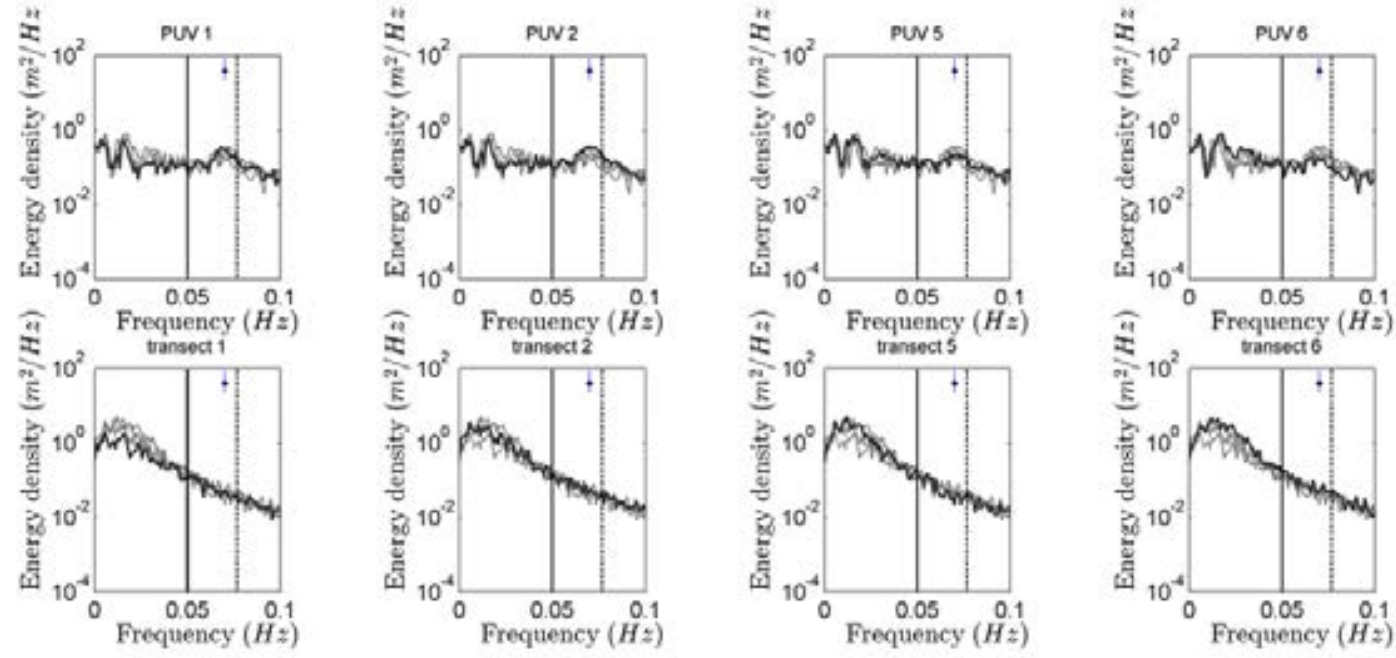

Figure 3. Spectre de densité d'énergie moyen au niveau de chaque capteur PUV/ transect (noir). Les courbes en gris représentent celui des autres transects. La barre d'intervalle de confiance à 95\% est également indiquée.

Ces observations sont cohérentes avec de précédentes observations dans des conditions similaires (RUESSINK et al., 1998 ; RUGGIERO et al., 2004 ; SENECHAL et al., 
2011a). La figure 3 montre également que les niveaux énergétiques observés au niveau des différents transects sont différents et qu’ils présentent un gradient longitudinal alors que les données en zone de surf ne semblent pas présenter de variabilité longitudinale. En particulier, on observe que la fréquence jusqu'à laquelle s'étend la zone de saturation du spectre est plus basse $(0.022 \mathrm{~Hz})$ au niveau des Transects T1 et T2 qu'au niveau des transects T5 et T6 $(0.033 \mathrm{~Hz})$. Afin de mieux caractériser ce gradient longitudinal, les hauteurs de jet de rive et de vagues en zone de surf ont été calculées à partir de l'équation 2.

\subsection{Gradient longitudinal des hauteurs de jet de rive}

Le tableau 1 représente les valeurs moyennes calculées à partir des valeurs extraites des 17 fenêtres temporelles. On observe très clairement que l'énergie du jet de rive est essentiellement contenue dans la bande infragravitaire avec un rapport $R_{I n} / R_{I g}$ inférieur à 0.35 pour l'ensemble des transects. Cette répartition est caractéristique des dynamiques de jet de rive associées à de faibles paramètres d'Iribarren en présence de conditions dissipatives (RUESSINK et al., 1998 ; RUGGIERO et al., 2004 ; BRINKKEMPER et al., 2013). Dans la zone de surf interne par contre, l'énergie est principalement dominée par l'énergie de la bande incidente.

Tableau 1. Hauteur moyenne (m) calculée selon l'équation 2 de jet de rive au niveau de chaque transect pour chacune des bandes fréquentielles (In : incidente, Ig :

Infragravitaire, $T$ : totale) et des vagues en zone de surf au niveau de chaque capteur PUV.

\begin{tabular}{lllllllll}
\hline & \multicolumn{7}{l}{ Zone de surf } & \multicolumn{7}{c}{ Jet de rive } \\
\cline { 2 - 9 } & PUV1 & PUV2 & PUV5 & PUV6 & Trans1 & Trans2 & Trans5 & Trans6 \\
\hline$R_{T}$ & 0.39 & 0.43 & 0.45 & 0.41 & 0.75 & 1.00 & 1.08 & 1.17 \\
$R_{I g}$ & 0.21 & 0.22 & 0.27 & 0.28 & 0.66 & 0.90 & 1.01 & 1.09 \\
$R_{I n}$ & 0.26 & 0.29 & 0.28 & 0.22 & 0.21 & 0.23 & 0.20 & 0.24 \\
$R_{I n} / R_{I g}$ & 1.25 & 1.32 & 1.02 & 0.79 & 0.32 & 0.27 & 0.20 & 0.22 \\
\hline
\end{tabular}

On observe qu'en zone de surf, le gradient longitudinal de hauteurs des vagues est faible ; l'utilisation de tests statistiques (Snedecor et Student) indique que celui-ci n'est pas statistiquement significatif. Au contraire, en zone de jet de rive, on observe un gradient statistiquement significatif de la hauteur de jet de rive. Celui-ci est essentiellement contraint par la composante infragravitaire du jet de rive qui varie d'un facteur 1.56 entre la plus basse valeur observée (Trans1) et la plus forte valeur observée (Trans6). Plusieurs pistes peuvent être proposées pour expliquer ce gradient longitudinal. Celui-ci peut en partie s'expliquer par la pente de plage. En effet, si on observe les valeurs d'Iribarren calculées au niveau des différents Transects (figure 2), on observe que celles-ci sont systématiquement plus élevées au niveau du transect T6 


\section{XIII ${ }^{\text {èmes }}$ Journées Nationales Génie Côtier - Génie Civil \\ Dunkerque, 2-4 juillet 2014}

qu'au niveau des Transects T1 et T2. Or RUGGIERO et al. (2004) avait montré que la composante infragravitaire était linéairement corrélée à la pente de plage. Toutefois la variation de pente de plage observée ici (au maximum un rapport de 1.2) ne peut pas à elle seule expliquer le gradient de hauteurs de jet de rive. De la même façon on observe un gradient des hauteurs moyennes calculées dans la bande infragravitaire en zone de surf (tableau 1, $R_{I g}$ ) qui est cohérent avec le gradient observé en zone de jet de rive mais d'amplitude plus faible (au maximum 1.3). De récentes études (GUEDES et al., 2012) ont également montré que les taux de dissipation des vagues ainsi que les processus de génération/libération d'harmoniques au passage d'une barre pouvaient expliquer en partie le gradient longitudinal de hauteurs de jet de rive.

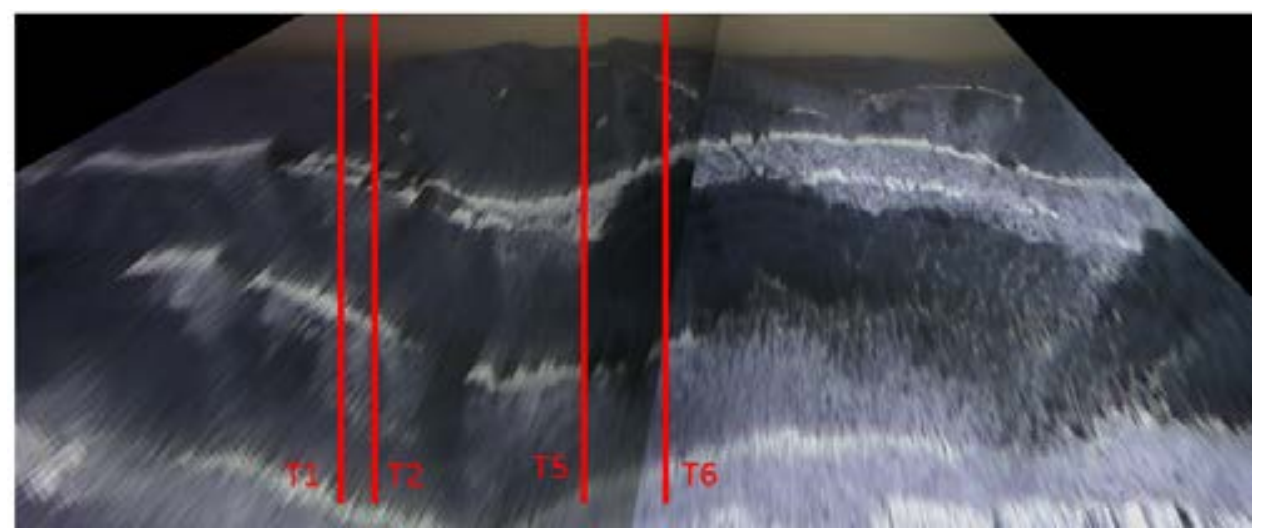

Figure 4. Image instantanée redressée. On observe la réfraction des crêtes de houle autour de la figure morphologique en bas de plage, ainsi que le gradient longitudinal du taux de dissipation d'énergie des vagues.

Toutefois dans leur étude, la dynamique du jet de rive était contrôlée par l'énergie incidente et non par l'énergie infragravitaire comme c'est le cas dans notre étude. La figure 4 illustre qualitativement le gradient longitudinal du taux de dissipation des vagues mais également la réfraction de celles-ci autour de la forme morphologique observée en bas de plage, concentrant l'énergie notamment au niveau du Transect T6. Des études sont actuellement en cours afin de mieux caractériser l'impact de ces processus sur le gradient longitudinal du jet de rive, incluant notamment l'extraction de transects supplémentaires.

\section{Conclusions}

L'analyse de données de jet de rive extraites à partir d'imagerie vidéo a permis de montrer qu'en présence de conditions de forçage stationnaires, la hauteur associée au jet de rive pouvait varier d'un facteur 1.56 sur une courte distance $(<250 \mathrm{~m})$. Cette variabilité ne peut à priori pas uniquement s'expliquer par les paramètres environnementaux incluant la hauteur des vagues au large mais également en zone de 
surf interne et la pente de plage. Ces résultats indiquent la limitation des formules paramétriques habituellement utilisées en ingénierie côtière afin de dimensionner les ouvrages côtiers et de prévoir les risques de submersion et érosion. Des analyses sont en cours afin de mieux évaluer le rôle de la figure morphologique présente en bas de plage sur le gradient observé de jet de rive.

\section{Remerciements}

Nous remercions V. Marieu pour son soutien logistique et son aide dans le développement des scripts d'analyse. Nous remercions également J. MacMahan pour la mise à disposition des données de capteurs de pression PUV e zone de surf interne.

\section{Références bibliographiques}

BRINKKEMPER, J.A., TORRES-FREYERMUTH, A., MENDOZA, E.T., RUESSINK, B.G. (2013). Parameterization of wave run-up on beaches in Yucatan Mexico: a numerical study. Proceeding of Coastal Dyamics 2013 - Arcachon - France, pp 225-234.

GUEDES R.M.C., BRYAN K.R., COCO G. (2012). Observations of alongshore variability of swash motions on an intermediate beach. Continental Shelf research, Vol. 48, pp 61-74. http://dx.doi.org/10.1016/j.csr.2012.08.022

GUZA R.T., THORNTON E.B. (1982). Swash oscillations on a natural beach. J. Geophys. Res., Vol. 87, pp 483-491. http://dx.doi.org/10.1029/JC087iC01p00483

RUESSINK B.G., KLEINHANS M.G., VAN DEN BEUKEL P.G.L. (1998). Observations of swash under highly dissipative conditions. J. Geophys. Res., Vol. 103, pp 3111-3118. http://dx.doi.org/10.1029/97JC02791

RUGGIERO P., HOLMAN R.A., BEACH R.A. (2004). Wave run-up on a high-energy dissipative beach. J. Geophys. Res., Vol. 109, C0625. http://dx.doi.org/10.1029/2003JC002160

SENECHAL N., COCO G., BRYAN K.R., HOLMAN R.A. (2011a). Wave runup during extreme wave conditions. J. Geophys. Res., Vol. 116(C7). http://dx.doi.org/10.1029/2010JC006819

SENECHAL et al. (2011b). The ECORS- Truc Vert'08 nearshore field experiment: presentation of a three-dimensional morphologic system in a macro-tidal environment during consecutive extreme storm conditions. Ocean Dynamics, Vol. 61(12), pp 20732098. http://dx.doi.org/10.1007/s10236-011-0472-x

STOCKDON H.F., HOLMAN R.A., HOWD P.A., SALLENGER JR. A.H. (2006). Empirical parameterizations of setup, swash and runup. Coast. Eng., Vol. 53, pp 573588. http://dx.doi.org/10.1016/j.coastaleng.2005.12.005 